\title{
Working memory modulates the perception of time
}

\author{
Yi Pan • Qian-Ying Luo
}

Published online: 29 November 2011

(C) Psychonomic Society, Inc. 2011

\begin{abstract}
Recent research has indicated that reentrant feedback from the contents of working memory can enhance neural representations and the perceptual strengths of matching stimuli in the visual field. However, whether the contents of working memory can also distort conscious experiences of perception remains unclear. Our present results show that the durations of perceptual stimuli matching the nontemporal representations in working memory tend to be perceived as longer than those of mismatching stimuli. This is the first demonstration that working memory can lead to distortions of time perception. Our findings are consistent with the ideas that the perceived duration of a stimulus depends on the magnitude of the neural responses to that stimulus in visual cortex and that there is a common system for representing both temporal and nontemporal magnitudes. We conclude that top-down modulation from the nontemporal contents of working memory distorts the perceptual experience of temporal duration.
\end{abstract}

Keywords Working memory - Time perception - Subjective duration $\cdot$ Magnitude

Working memory (WM) and perception are commonly thought to interact with each other in different ways. Important aspects of such interactions are the influences of WM representations on attentional selection and perceptual processing (for recent reviews, see Pan, 2010; Soto, Hodsoll, Rotshtein, \& Humphreys, 2008). According to the

Y. Pan $(\bowtie) \cdot$ Q.-Y. Luo

Department of Psychology, Hangzhou Normal University,

Hangzhou 310036, China

e-mail: panyirich@zju.edu.cn biased competition of attention, the contents of WM may provide top-down feedback signals from higher cortical areas (e.g., prefrontal cortex) to visual cortex, enhancing the activity of neurons that perform perceptual analysis of stimuli matching the WM contents and suppressing neuronal responses to mismatching stimuli (Desimone, 1996; Desimone \& Duncan, 1995). A number of studies have provided strong neurophysiological evidence for such WM-driven modulation of neural signals in early visual areas (e.g., Chelazzi, Miller, Duncan, \& Desimone, 1993; Miller \& Desimone, 1994; Soto, Humphreys, \& Rotshtein, 2007). Moreover, converging behavior evidence has recently emerged showing that a target presented with distractors can be identified more accurately when it matches the current contents in WM (Soto \& Humphreys, 2006; Soto, Wriglesworth, Bahrami-Balani, \& Humphreys, 2010). This implies that enhanced neural responses to stimuli matching the contents of WM in visual cortex render the corresponding perceptual strengths of those stimuli more intense, and therefore improve behavior performance.

It is clear that our perceptual experiences usually do not have a direct, precise mapping onto the external world. Instead, perception is prone to distortions depending on the influences of many factors. However, there has as yet been little indication that top-down feedback from the contents of WM could lead to perception distortions. For example, Turatto, Vescovi, and Valsecchi (2008) showed that reentrant feedback from the contents of WM does not alter the perceived motion speed of memory-matching objects in the visual field. Likewise, Pan and Cheng (2011) found no evidence suggesting that visual awareness can be accelerated via top-down modulation from WM. Nevertheless, because a stimulus has many different properties, and the perceptual mechanisms of those stimulus properties are also very different from one another, the null effects of WM in the 
aforementioned literature would not necessarily generalize to other aspects of perceptual processing.

Previous studies regarding WM effects on perception have merely focused on nontemporal properties of a perceptual stimulus, such as color and shape. However, every stimulus also has a temporal property, namely duration. Estimation of duration constitutes a fundamental aspect of human cognitive functioning. Despite the critical role of duration perception, a large body of evidence has suggested that many nontemporal factors can modulate subjective duration (e.g., Lu, Hodges, Zhang, \& Zhang, 2009; Matthews, 2011; Matthews, Stewart, \& Wearden, 2011; Pariyadath \& Eagleman, 2008; Yeshurun $\&$ Marom, 2008). For example, the duration of a stimulus with a larger size tends to be perceived as longer than that of a stimulus with a smaller size (Ono \& Kawahara, 2007; Xuan, Zhang, He, \& Chen, 2007). Motivated by these observations, here we asked for the first time whether top-down feedback from WM representations can influence perceived duration. We conjectured that the subjective duration of a stimulus matching the contents of WM might be longer than that of a mismatching stimulus. This assumption was made on the basis of a time perception framework proposed by Eagleman and Pariyadath (2009). These authors assumed that the subjective duration of a brief stimulus depends on the magnitude of neural activity triggered by that stimulus, with increased neural responses also leading to duration elongations. That is to say, the duration of a stimulus is directly represented as the firing rate of neurons selective for that stimulus in early visual areas. If this framework is really correct, we should expect that WM representations would prolong the perceived durations of matching stimuli, because convincing evidence has suggested that top-down feedback from the contents of WM can intensify neural activity to memory-matching stimuli in visual cortex (e.g., Soto et al., 2007).

\section{Experiment 1}

The purpose of the present study was to test the hypothesis that top-down feedback from the nontemporal information actively held in WM might affect the perceived duration of matching stimuli in the visual field. During the retention interval of a WM task, observers had to make a judgment regarding which of the two colored circles presented in succession at the center of the screen had a longer (or shorter) duration, with one circle matching the color of the memory cue presented at the beginning of the trial and the other mismatching the cue. The objective durations of both circles were always different from each other on every trial. The primary dependent measure was duration judgment accuracy. We predicted that WM would prolong subjective duration, so that there would be more duration judgment errors when the memory-matching circle had a shorter objective duration than when it had a longer objective duration.

\section{Method}

Participants A group of 30 naive students at Hangzhou Normal University participated for partial course credit. All of them reported having normal or corrected-to-normal vision.

Stimuli The visual stimuli were colored squares (each $3.5^{\circ} \times$ $3.5^{\circ}$ ) in the memory task and colored circles (each $3^{\circ} \times 3^{\circ}$ ) in the paired duration judgment task. The color of each shape was chosen randomly from a set of five colors (red, green, blue, yellow, and cyan). The paired durations of the circles were 600/750, 650/812, 700/875, and 750/937 ms (all conforming to a shorter/longer ratio of $1 / 1.25$ ), which were the same as those used in Xuan, Zhang, He, and Chen's (2007) study. All stimuli were presented on a gray background at a viewing distance of $57 \mathrm{~cm}$.

Procedure and design Participants initiated each trial by pressing the space bar. Each trial began with the display of a white central fixation cross $\left(0.2^{\circ} \times 0.2^{\circ}\right)$ for $1,000 \mathrm{~ms}$. Then a colored square was presented at the center of the screen for $600 \mathrm{~ms}$. Here, participants were instructed to memorize the color of the square and to keep it in mind throughout the entire trial. After a delay of $200 \mathrm{~ms}$, two different colored circles were successively presented at the center of the screen, with an interstimulus interval of $200 \mathrm{~ms}$. Immediately after the offset of the second circle, participants were asked to make an unspeeded judgment regarding which of the two circles was presented for a longer (or shorter) duration by pressing one of two buttons. A memory test followed $500 \mathrm{~ms}$ after the duration judgment was completed. Here, a colored square appeared at the center of the screen, and participants were asked to indicate by buttonpress whether or not it had the same color as the memory cue (see Fig. 1).

The memory test item and the memory cue shared the same color on half of the trials. The paired circles were always different from each other in color on each trial, with one circle matching the color of the memory cue and the other mismatching the color of the cue. The four duration pairs occurred with equal probabilities (i.e., $25 \%$ of all trials for each pair) and were randomized across trials. The memory-matching circle had a longer duration than the mismatching one on half of the trials. Also, the memorymatching circle was presented earlier than the mismatching one on half of the trials. These two factors varied randomly across trials. To eliminate the potential impact of a response bias for the memory-matching circle on the results, half of the participants were asked to indicate which of the two circles had a longer duration, and the other half were asked to indicate 


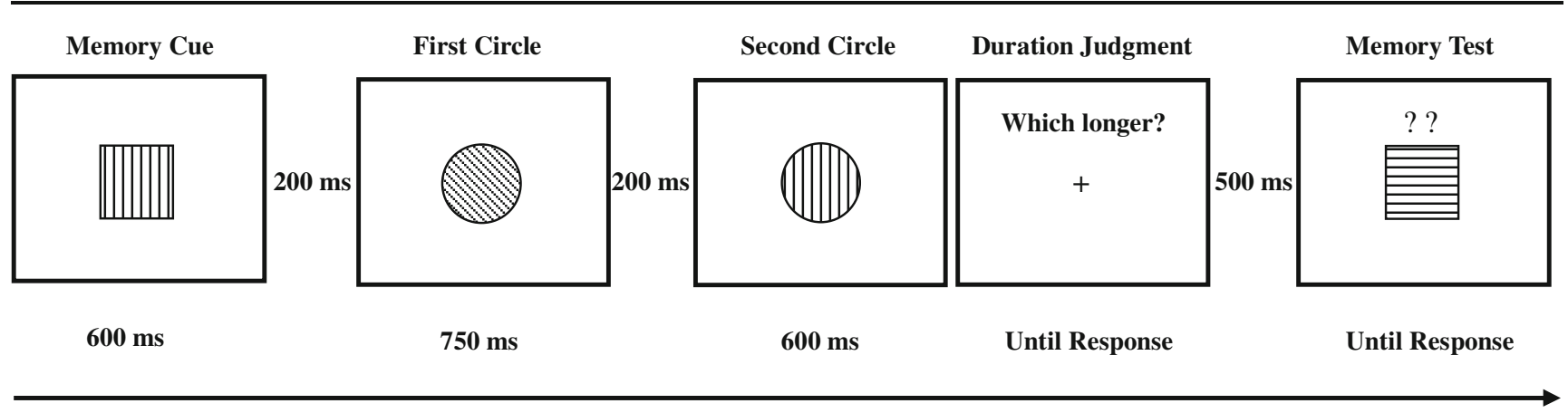

Fig. 1 A sample trial in Experiment 1, in which the memory-matching circle was presented for $600 \mathrm{~ms}$ (a shorter duration) and the mismatching circle was presented for $750 \mathrm{~ms}$ (a longer duration). The different patterns represent different colors

which had a shorter duration. Participants were never asked to report the color of a circle, but simply to indicate the temporal order of the onset of the circle (first vs. second) that was presented for a longer (or shorter) duration. A total of 40 practice trials were followed by 240 experimental trials.

\section{Results and discussion}

Errors in the memory task averaged $9.6 \%$ and did not vary according to whether or not the memory-matching circles were presented for a shorter duration than mismatching ones $(9.55 \%$ vs. $9.58 \%), F(1,29)=0.002, p=.962$. To determine the effect of WM on duration perception, analyses of the duration judgment data included only trials on which memory responses were correct. As is shown in Fig. 2A, there were more duration judgment errors when memory-matching circles had a shorter duration than mismatching ones $(M=$ $30 \%$ ), as compared to when memory-matching circles had a longer duration than mismatching ones $(M=26 \%), F(1,29)=$ $8.707, p=.006, \eta_{\mathrm{p}}^{2}=.231$. There was no sign of a speedaccuracy trade-off, because median RTs in the duration judgment task did not differ significantly between the two conditions (785 vs. $791 \mathrm{~ms}$ ), $F(1,29)=0.05, p=.825$. The results suggest that the durations of stimuli matching the current contents of WM tended to be perceived as longer than those of mismatching stimuli, leading to more duration judgment errors when memory-matching stimuli actually had a shorter duration than the mismatching stimuli.

\section{Experiment 2}

The aim of this experiment was to examine whether the effect of WM on subjective duration observed in Experiment 1 was indeed due to the active maintenance of the cue in WM. Would mere exposure to the color cue without WM processing be sufficient to prolong the perceived duration of matching stimuli?

\section{Method}

This was similar to the method of Experiment 1, with the following exceptions. A new group of 20 volunteers from the same pool participated. The observers were asked to attend to the cue, but they did not need to memorize it, and there was no memory test at the end of the trial. A go/no-go procedure was used to allow the cue to be processed without being held in WM. Observers were asked to complete the duration judgment task when the cue was a colored square, on $80 \%$ of the trials, whereas they should not carry out the duration judgment task when the cue was a black square, on the remaining $20 \%$ of the

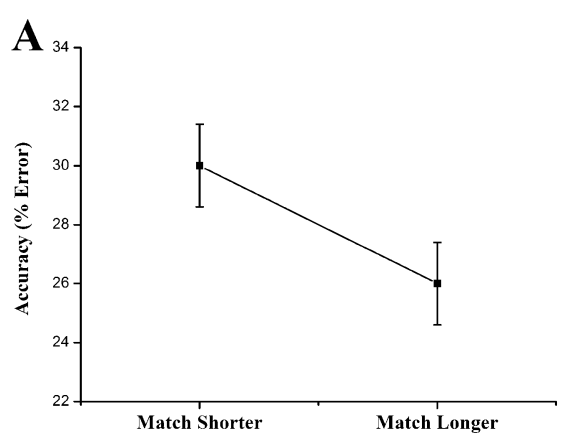

Fig. 2 Duration judgment accuracy in Experiment 1 (A), 2 (B), and 3 (C), displayed as a function of whether or not the circles that matched the cues were presented for the shorter duration. Error bars
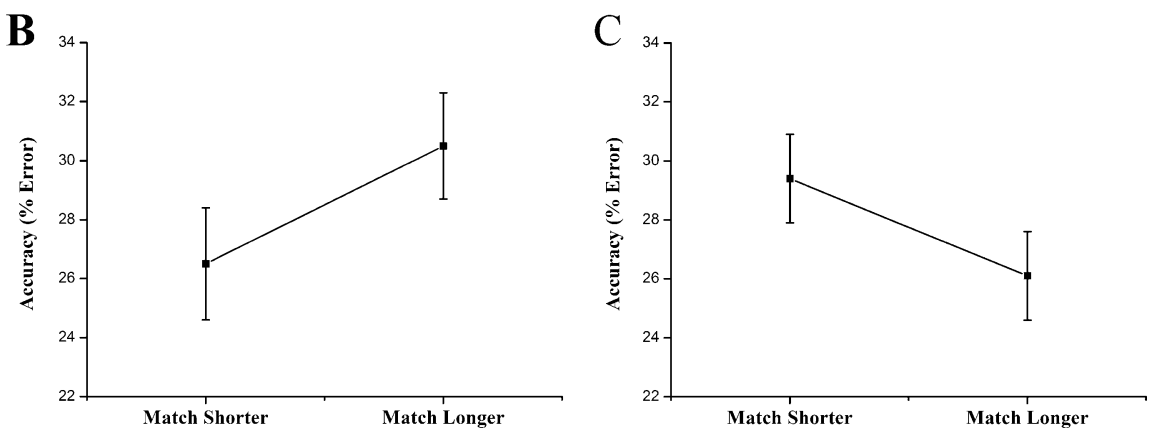

represent within-subjects $95 \%$ confidence intervals, as described by Loftus and Masson (1994) 
trials. (In the latter case, the observers were asked to end the trial by pressing the space bar.) The participants performed 30 practice trials, followed by 300 experimental trials.

\section{Results and discussion}

The observers performed appropriately as requested on catch trials, where the cue was a black square $(98.1 \%$ correct on average). Duration judgment errors averaged $28.5 \%$ and varied significantly according to whether or not matching circles were presented for a shorter duration than mismatching ones, $F(1,19)=5.469, p=.03, \eta_{\mathrm{p}}^{2}=.223$. As is shown in Fig. $2 \mathrm{~B}$, in contrast to the finding from Experiment 1, here there were fewer duration judgment errors when matching circles had a shorter duration $(M=26.5 \%)$ than when they had a longer duration $(M=30.5 \%)$. There was no sign of a speed-accuracy trade-off, because median RTs did not differ significantly between the two conditions ( 527 vs. $547 \mathrm{~ms}$ ), $F(1,19)=1.499, p=.236$. This pattern of results suggests that priming an object's representation without WM requirements did not prolong the perceived duration of matching stimuli, although the prime was perceptually identified. Instead, mere exposure to the color cue without WM processing shortened the duration of stimuli that matched the perceptual cue, leading to more duration judgment errors when matching stimuli actually had a longer duration than the mismatching stimuli. Thus, the prolonged perceivedduration effect observed in Experiment 1 could not be attributed to the mechanism of perceptual priming. This was further confirmed by a comparison of duration judgment errors between Experiments 1 and 2, which yielded a significant interaction effect, $F(1,48)=13.64, p=.001$, $\eta_{\mathrm{p}}{ }^{2}=.221$. We suggest that object representations need to be actively maintained in WM to prolong the perceived duration of matching stimuli in the visual field.

\section{Experiment 3}

The final experiment sought to replicate the WM effect on subjective duration in Experiment 1 with verbal instead of visual memory cues. Would a relatively abstract match between a memory cue and a perceptual stimulus based on common conceptual representations be sufficient to prolong the perceived duration of that stimulus? Furthermore, this experiment provided another control of bottom-up repetition priming, because here no exact physical features were shared between a memory cue and a perceptual stimulus.

\section{Method}

This was virtually identical to the method of Experiment 1, with the following exceptions. The colors of the paired circles in the duration judgment task were never cyan. The memory cues were four Chinese characters (which indicated "red," "blue," "yellow," and "green," respectively), presented for $1,000 \mathrm{~ms}$ at the beginning of the trial, and the memory test items were four English words (semantically matched to the Chinese words); all of the word stimuli were printed in black. This manipulation would force observers to complete the memory task based on the congruency of the semantic meanings rather than the visual forms of the memory cue and the memory test item. Also, to further ensure that the meaning of a verbal cue would really be identified and held in WM, observers were explicitly asked to verbalize the cue and to retain it in mind throughout the trial. A group of 20 native Chinese university students participated in this experiment. They were all expert readers of English. The participants performed 48 practice trials, followed by 192 experimental trials.

\section{Results and discussion}

Errors in the memory task averaged $4.4 \%$ and did not vary according to whether or not the memory-matching circles were presented for a shorter duration than the mismatching ones $(4.79 \%$ vs. $3.96 \%), F(1,19)=1.773, p=.199$. As in Experiment 1, analyses of the duration judgment data included only trials on which the memory responses were correct. As is shown in Fig. 2C, more duration judgment errors occurred when the memory-matching circles had a shorter duration $(M=29.4 \%)$ than when they had a longer duration $(M=26.1 \%), F(1,19)=5.247, p=.034, \eta_{\mathrm{p}}{ }^{2}=$ .216. There was no sign of a speed-accuracy trade-off, because median RTs in the duration judgment task did not vary significantly between the two conditions (730 vs. $733 \mathrm{~ms}), F(1,19)=0.043, p=.838$. These results indicate that the durations of visual stimuli matching the verbal contents of WM tended to be perceived as longer than those of mismatching stimuli.

\section{General discussion}

Our present study establishes a modest but reliable distortion effect of WM on time perception. Specifically, we demonstrated that visual stimuli can appear longer in duration when they match the nontemporal representations in WM, regardless of whether such a match is established at an exact physical level (Exp. 1) or at an abstract conceptual level (Exp. 3). Such duration elongation effects cannot be attributed to the mechanism of bottom-up perceptual priming from the presentation of a memory cue, because mere exposure to the cue without WM processing actually shortened apparent durations (Exp. 2). Moreover, it is unlikely that the prolonged subjective duration effects were 
induced by spatial attention shifts driven by the WM representations. In the present experiments, all stimuli were presented successively in the center of the screen, and observers had to maintain their attention at fixation without shifting it to other locations. Thus, despite the evidence that WM representations can guide attention to the location of matching stimuli in the visual field (Soto et al., 2008), we believe that such spatial attention shifts did not occur in the present study. If any WM effect on attention existed here, that effect could be dimension-based, WM-driven attentional selection (Pan, Xu, \& Soto, 2009). Specifically, because observers were asked to hold a color cue in WM, their attention could have been guided to select the color dimension of the memory-matching circle during the duration judgment task, probably to refresh their memory of the color cue to help them perform the subsequent memory test. However, because limited attentional capacity is shared for processing both temporal and nontemporal stimulus properties (Thomas \& Weaver, 1975), processing the color of a stimulus should direct attention away from the temporal aspect of that stimulus, rendering the subjective duration actually shorter rather than longer (Macar, Grondin, \& Casini, 1994). As such, our results suggest that the perceived duration of a stimulus can be modulated directly by WM, without any attentional mediation.

The present findings strongly support the idea that the perceived duration of a stimulus correlates with the intensity of the neural responses to that stimulus, with intensified neural activity leading to prolonged subjective duration (Eagleman \& Pariyadath, 2009). We showed that holding a stimulus in WM can lead to a longer subjective duration for that stimulus, whereas mere perceptual repetition without WM processing actually shortens the apparent duration. Such duration distortions can be well understood in terms of the different neural mechanisms of top-down WM effects on perception and bottom-up perceptual repetition. As we noted in the introduction, strong evidence has shown that top-down feedback from WM representations enhances the neural activity selective for memory-matching stimuli in visual cortex (e.g., Chelazzi et al., 1993; Miller \& Desimone, 1994; Soto et al., 2007). By contrast, repetition suppression underlies the neural mechanism of bottom-up perceptual repetition, in which neural responses to repeated stimuli are diminished (Desimone, 1996; Soto et al., 2007). Therefore, our results corroborate the view that time perception is based on the magnitude of neural activity. Furthermore, because Eagleman and Pariyadath (2009) suggested that "the perception of time may be related to very low-level properties of neurons, rather than a highly cognitive algorithm" (p. 1844), the present findings imply that their framework should be refined to account for the influence of high-order cognitive processes such as WM. We suggest that higher cortical areas, via top-down modulation of neuronal activity in sensory cortex, contribute significantly to time perception.

Our results also support the notion of a common representational system for both temporal and nontemporal magnitudes (e.g., Xuan et al., 2007). According to the theory of magnitude proposed by Walsh (2003), there must be a general substrate in the human brain (e.g., inferior parietal cortex) for representing magnitudes in different dimensions, such as space and time. Different magnitudes may be represented by a common property in such a generalized magnitude system, so that there is an intrinsic association between magnitudes in the different dimensions of a stimulus. In line with this theory, the present study provides additional evidence suggesting that a common representation exists for magnitudes of intensity and time, with an increased intensity of nontemporal stimulus attributes via top-down modulation from WM leading to longer perceived temporal duration.

The present findings challenge an "uncertainty reduction" account for WM effects on perception (Cosman \& Vecera, 2011). According to this account, the contents of WM do not modulate perception, but rather operate postperceptually to reduce the uncertainty of a target's location by prioritizing the memory-matching stimulus in visual search. The uncertainty reduction account predicts that WM effects should occur only in conditions in which there is uncertainty regarding the search target's location, and that no WM effects should occur when the target's location is known with certainty. These predictions appear to be supported by the findings of Cosman and Vecera showing that WM effects on visual search occurred when the search array consisted of more than one item, and disappeared when the search array consisted of only one item. However, we suggest that WM does affect perception, and that the reason why Cosman and Vecera did not observe WM effects on the perceptual sensitivity of a search target presented in isolation might be a lack of power of their task paradigm. In the present study, using a paradigm combining WM and time perception, we showed that although perceptual stimuli were always presented at central fixation, and therefore their location was known with absolute certainty, WM could still exert a significant influence on perception. Thus, our results provide strong evidence going against the uncertainty reduction account: WM can modulate the perception of a stimulus presented at a location that is known with certainty.

In conclusion, the present findings indicate that topdown modulation from the nontemporal contents of WM prolongs the perceived durations of visual matching stimuli. Consistent with the broader notion that interactions occur between WM and perception, the present study provides a demonstration that WM can distort our perceptual experiences of time. 
Author note This research was supported by grants from the National Natural Science Foundation of China (31000462), the Zhejiang Provincial Social Science Foundation (10CGJY11YB), and the Qinshen Research Project of Hangzhou Normal University.

\section{References}

Chelazzi, L., Miller, E. K., Duncan, J., \& Desimone, R. (1993). A neural basis for visual search in inferior temporal cortex. Nature, 363, 345-347. doi:10.1038/363345a0

Cosman, J. D., \& Vecera, S. P. (2011). The contents of visual working memory reduce uncertainty during visual search. Attention, Perception, \& Psychophysics, 73, 996-1002. doi:10.3758/ s13414-011-0093-y

Desimone, R. (1996). Neural mechanisms for visual memory and their role in attention. Proceedings of the National Academy of Sciences, 93, 13494-13499.

Desimone, R., \& Duncan, J. (1995). Neural mechanisms of selective visual attention. Annual Review of Neuroscience, 18, 193-222. doi:10.1146/annurev.ne.18.030195.001205

Eagleman, D. M., \& Pariyadath, V. (2009). Is subjective duration a signature of coding efficiency? Philosophical Transactions of the Royal Society B, 364, 1841-1851.

Loftus, G. R., \& Masson, M. E. J. (1994). Using confidence intervals in within-subject designs. Psychonomic Bulletin \& Review, 1, 476-490. doi:10.3758/BF03210951

Lu, A., Hodges, B., Zhang, J., \& Zhang, J. X. (2009). Contextual effects on number-time interaction. Cognition, 113, 117-122. doi:10.1016/j.cognition.2009.07.001

Macar, F., Grondin, S., \& Casini, L. (1994). Controlled attention sharing influences time estimation. Memory \& Cognition, 22, 673-686. doi:10.3758/BF03209252

Matthews, W. J. (2011). How do changes in speed affect the perception of duration? Journal of Experimental Psychology: Human Perception and Performance, 37, 1617-1627. doi:10.1037/a0022193

Matthews, W. J., Stewart, N., \& Wearden, J. H. (2011). Stimulus intensity and the perception of duration. Journal of Experimental Psychology: Human Perception and Performance, 37, 303-313. doi:10.1037/a0019961

Miller, E. K., \& Desimone, R. (1994). Parallel neuronal mechanisms for short-term memory. Science, 263, 520-522. doi:10.1126/ science. 8290960
Ono, F., \& Kawahara, J.-I. (2007). The subjective size of visual stimuli affects the perceived duration of their presentation. Perception \& Psychophysics, 69, 952-957. doi:10.3758/BF03193932

Pan, Y. (2010). Content-based working memory-driven visual attention [in Chinese]. Advances in Psychological Science, 18, 210-219.

Pan, Y., \& Cheng, Q.-P. (2011). Do working memory-driven attention shifts speed up visual awareness? Attention, Perception, \& Psychophysics.. doi:10.3758/s13414-011-0193-8

Pan, Y., Xu, B., \& Soto, D. (2009). Dimension-based working memorydriven capture of visual selection. Quarterly Journal of Experimental Psychology, 62, 1123-1131. doi:10.1080/17470210802624353

Pariyadath, V., \& Eagleman, D. M. (2008). Brief subjective durations contract with repetition. Journal of Vision, 8(16), 11:1-6. doi:10.1167/8.16.11

Soto, D., Hodsoll, J., Rotshtein, P., \& Humphreys, G. W. (2008). Automatic guidance of attention from working memory. Trends in Cognitive Sciences, 12, 342-348. doi:10.1016/j.tics.2008.05.007

Soto, D., \& Humphreys, G. W. (2006). Seeing the content of the mind: Enhanced awareness through working memory in patients with visual extinction. Proceedings of the National Academy of Sciences, 103, 4789-4792. doi:10.1073/pnas.0510718103

Soto, D., Humphreys, G. W., \& Rotshtein, P. (2007). Dissociating the neural mechanisms of memory-based guidance of visual selection. Proceedings of the National Academy of Sciences, 104, 17186-17191. doi:10.1073/pnas.0703706104

Soto, D., Wriglesworth, A., Bahrami-Balani, A., \& Humphreys, G. W. (2010). Working memory enhances visual perception: Evidence from single detection analysis. Journal of Experimental Psychology: Learning, Memory, and Cognition, 36, 441-456. doi:10.1037/ a 0018686

Thomas, E. A. C., \& Weaver, W. B. (1975). Cognitive processing and time perception. Perception \& Psychophysics, 17, 363-367. doi:10.3758/BF03199347

Turatto, M., Vescovi, M., \& Valsecchi, M. (2008). On altering motion perception via working memory-based attention shifts. Journal of Vision, 8(5), 11:1-13. doi:10.1167/8.5.11

Walsh, V. (2003). A theory of magnitude: Common cortical metrics of time, space and quantity. Trends in Cognitive Sciences, 7, 483488. doi:10.1016/j.tics.2003.09.002

Xuan, B., Zhang, D., He, S., \& Chen, X. (2007). Larger stimuli are judged to last longer. Journal of Vision, 7(10), 2:1-5. doi:10.1167/7.10.2

Yeshurun, Y., \& Marom, G. (2008). Transient spatial attention and the perceived duration of brief visual events. Visual Cognition, 16, 826-848. doi:10.1080/13506280701588022 